\title{
A gene encoding a family with sequence similarity 84 , member A (FAM84A) enhanced migration of human colon cancer cells
}

\author{
TAKAAKI KOBAYASHI ${ }^{1,3}$, TADAHIKO MASAKI ${ }^{3}$, MASANORI SUGIYAMA $^{3}$, \\ YUTAKA ATOMI $^{3}$, YOICHI FURUKAWA ${ }^{2}$ and YUSUKE NAKAMURA ${ }^{1}$ \\ ${ }^{1}$ Laboratory of Molecular Medicine, Human Genome Center, ${ }^{2}$ Promotion of Genome-Based Medicine Project, \\ Institute of Medical Science, The University of Tokyo, Tokyo 108-8639; ${ }^{3}$ Department of Surgery, \\ Kyorin University School of Medicine, Tokyo 181-8611, Japan
}

Received January 3, 2006; Accepted February 23, 2006

\begin{abstract}
Using genome-wide cDNA microarray analysis, we identified a number of genes whose expression was upregulated frequently in colorectal cancer. One of these was a gene termed FAM84A that was not expressed in any of the 23 normal tissues examined except the testis. Although immunocytochemical staining revealed localization of FAM84A protein in the subcellular membrane region, the staining was limited to the region lacking attachment with neighboring cells. In addition, we found that exogenous FAM84A expression increased cell motility in NIH3T3 cells, and that phosphorylation of serine 38 of FAM84A was associated with morphology of cells. Our results indicate a possibility that up-regulation of FAM84A plays a critical role in progression of colon cancer.
\end{abstract}

\section{Introduction}

Colorectal carcinoma (CRC) is one of the most common cancers worldwide. Although treatment of colorectal cancer has been improved in the last 15 years in the United States, it is still the second leading cause of cancer-related deaths ( $~ 57,000$ deaths in 2004) (1). In Japan, the incidence of colorectal cancer has been significantly increasing probably due to a change of life style and dietary habits, and more than 35,000 deaths were caused by CRC $(2,3)$. Early detection is the best way to avoid cancer death and, in fact, the 5-year survival of patients at stage I is as high as $\sim 80-95 \%$ (4). Despite improvements in diagnosis and treatment, the 5-year survival of patients at stage III or IV still remains as low as $25-60 \%$ and $0-7 \%$, respectively (4). Therefore, development of a novel therapeutic strategy for patients with advanced disease is required to further improve the prognosis. For this

Correspondence to: Dr Yusuke Nakamura, Laboratory of Molecular Medicine, Institute of Medical Science, The University of Tokyo, 4-6-1 Shirokanedai, Minato-ku, Tokyo 108-8639, Japan

E-mail: yusuke@ims.u-tokyo.ac.jp

Key words: FAM84A, colon cancer, migration purpose, it is essential to better understand the molecular mechanisms of colorectal carcinogenesis.

Colorectal carcinogenesis has been shown to involve accumulation of multiple genetic alternations including genetic changes in tumor suppressor genes and/or oncogenes (5-7), and epigenetic changes such as alteration of DNA methylation or splicing (8-10). These events result in loss of tumor suppressing function, deregulation of transcriptional control, and then enhancement of cell proliferation. Although recent studies have disclosed a number of genes associated with tumor growth or transformation, the mechanisms of invasion and metastasis largely remain unresolved. When tumor stays locally, it is curable by surgical resection. However, once malignant cells invade and metastasize to distant organs, the possibility to cure the disease by surgical treatment alone is significantly reduced. It is well known that invasion and migration of cancer cells are governed by a number of extracellular, transmembrane, and intracellular components that cause dissociation of cellcell adhesion, remodeling of extracellular matrices (ECM), increase of cellular motility, and morphological changes (11).

To clarify the molecular mechanisms underling colorectal tumors, and find novel therapeutic targets for better treatment, we have been analyzing expression profiles of CRC using cDNA microarray (12). Among the genes up-regulated in the cancer tissues, we focused, in this study, on FAM84A (family with sequence similarity 84 , member A). Transwell assay and wound-healing experiment showed that cells stably expressing FAM84A had increased cellular motility compared to control cells. These data suggest that elevated expression of FAM84A may be involved in invasion and/or metastasis of colon cancer cells.

\section{Materials and methods}

Cell lines and clinical tissue. Human colon cancer cell lines DLD1, HCT15, HCT116, HT29, SW948, SW480, cervical cancer line HeLa and fetal kidney cell line HEK293 were obtained from the American Type Culture Collection (ATCC, Rockville, MD). Human colon cancer cell line SNU C4 was obtained from Korean cell line bank (KCLB, Seoul, Korea). LS174T and RKO cells were gifts from Drs H. Fukamachi (Tokyo Medical and Dental University, Japan) and C.C. Harris (NCI, Washington DC), respectively. All of these cells were 
cultured as monolayers in appropriate media, as follows: Leibovitz's L-15 (Invitrogen, Carlsbad, CA) for SW948 and SW480; RPMI-1640 (Sigma-Aldrich Corporation, St. Louis, MO) for SNU C4, DLD1, HCT116, and HT29; Dulbecco's modified Eagle's medium (Sigma) for RKO and HEK293; Eagle's minimum essential medium (Sigma) for HeLa. Each medium was supplemented with $10 \%$ fetal bovine serum (Cansera, Ontario, Canada) and 1\% antibiotic/antimycotic solution (Sigma). SW948 and SW480 cells were maintained at $37^{\circ} \mathrm{C}$ in an atmosphere of humidified air without $\mathrm{CO}_{2}$, while other cells were maintained with $5 \% \mathrm{CO}_{2}$. Cancerous tissue and corresponding non-cancerous mucosa were obtained from 24 patients during surgery, after obtaining written informed consent.

Semiquantitative $R T-P C R$. Total RNA was extracted from culture cells or clinical tissue using TRIzol reagent according to the manufacturer's instructions (Invitrogen, Carlsbad, CA). Extracted RNA was treated with DNaseI (Roche Diagnostics, Mannheim, Germany) and reversely transcribed to singlestranded cDNAs using oligo(dT) $)_{12-18}$ primer with Superscript II reverse transcriptase (Invitrogen). We prepared appropriate dilutions of each single-stranded cDNA for subsequent PCR amplification by using $G A P D H$ as a quantitative control. Primer sequences were as follows: 5'-ACAACAGCCTCAAGATC ATCAG-3' and 5'-GGTCCACCACTGACACGTTG-3' for GAPDH, and 5'-ATTCGGCTCGGGGTAGAG-3' and 5'-TCT TCCTCATCATCCGAGAA-3' for FAM84A. All of the amplification reactions were preceded by initial denaturation at $94^{\circ} \mathrm{C}$ for $2 \mathrm{~min}$, followed by 20 (for $G A P D H$ ) or 36 cycles (for FAM84A) at $94^{\circ} \mathrm{C}$ for $30 \mathrm{sec}, 60^{\circ} \mathrm{C}$ for $30 \mathrm{sec}$, and $72^{\circ} \mathrm{C}$ for $30 \mathrm{sec}$ on a GeneAmp PCR system 9700 (PE Applied Biosystems, Foster, CA).

Northern blotting. Human multiple-tissue blots (BD Bioscience, Palo Alto, CA) were hybridized with a [ $\left.{ }^{32} \mathrm{P}\right]$-labeled PCR product of FAM84A. The probe was prepared by RT-PCR using a set of primers, 5'-ATTCGGCTCGGGGTAGAG-3' and 5'-TCTTCCTCATCATCCGAGAA-3', and subsequent random-oligonucleotide labeling with $\left.{ }^{32} \mathrm{P}\right]-\mathrm{dCTP}$ using a Mega Label kit (Amersham Biosciences, Buckinghamshire, UK). Prehybridization, hybridization, and washing were performed according to the supplier's recommendations. The blots were autoradiographed with intensifying screens at $-80^{\circ} \mathrm{C}$ for 14 days.

Immunoblot and immunocytochemical analyses with antiFAM84A antibody. The entire coding region of FAM84A was amplified using a set of primers, 5'-GATGAATT CACCTCATTGATGGGC-3' and 5'-GATCTCGAGCTCCT TGTCGTCC-3', and subsequently cloned into an appropriate cloning site of the pET28 vector (Novagen, Madison, WI) to construct plasmids expressing His-tagged FAM84A protein. The recombinant protein was expressed in Escherichia coli, the BL21-CodonPlus (DE3)-RIL strain (Stratagene, La Jolla, CA), and purified using TALON Superflow metal affinity resin (BD Bioscience) according to the manufacturer's protocol. The protein was inoculated into rabbits, and the immunized sera were purified on affinity columns using the standard method. We extracted proteins from tissues and cell lines using $0.1 \%$
RIPA-like buffer containing $50 \mathrm{mM}$ Tris- $\mathrm{HCl}(\mathrm{pH} 7.5)$, $250 \mathrm{mM} \mathrm{NaCl}, 0.1 \% \mathrm{SDS}$, and $0.5 \%$ NP40 with Protease inhibitor cocktail set III (Calbiochem, La Jolla, CA). Western blot analysis was carried out with polyclonal antibody against FAM84A as the primary antibody and HRP-conjugated antirabbit $\operatorname{IgG}$ (Amersham Bioscience) as the secondary antibody for the ECL detection system (Amersham Bioscience). Cultured cells on chamber slides were fixed with PBS containing $4 \%$ paraformaldehyde for $15 \mathrm{~min}$, then rendered permeable with PBS containing $0.1 \%$ Triton X-100 for $2.5 \mathrm{~min}$ at room temperature. The cells were covered with $3 \%$ BSA in PBS for $1 \mathrm{~h}$ at room temperature to block non-specific hybridization, and subsequently incubated with anti-FAM84A and anti-E-cadherin (Takara, Kyoto, Japan) antibodies. The reaction was visualized after incubation with anti-rabbit IgG antibody conjugated with Alexa Fluor 488 (Invitrogen) and anti-mouse IgG antibody or phalloidin conjugated with Alexa Fluor 594 (Invitrogen). Nuclei were counterstained with 4', 6'-diamidine-2'-phenylindole dihydrochloride (DAPI).

$\lambda$-phosphatase assay and establishment of stable NIH3T3 cells that express FAM84A. The entire coding region of FAM84A was amplified using a set of primers, 5'-GATGAATTCACC TCATTGATGGGC-3' and 5'-GATCTCGAGCTCCTTGTC GTCC-3', and subsequently cloned into an appropriate cloning site of pFLAG-CMV5 vector (Sigma) to generate plasmids expressing FLAG-tagged FAM84A protein. Lysates from HEK293 cells transfected with pFLAG-CMV-FAM84A were treated with or without $\lambda$-phosphatase (New England Biolabs, Beverly, MA). Plasmids expressing mutant FAM84A were prepared by PCR using pFLAG-CMV-FAM84A as a template with QuikChange II site-directed mutagenesis kit according to the supplier's recommendations (Stratagene). The sequences of oligonucleotide primers were 5'-GTTGCCTACTTCTTCG CGGATGATGAG-3' and 5'-CTCATCATCCGCGAAGAAG TAGGCAAC-3' for FAM84A-S38A, and 5'-CTGCCCGGA GGCCCCCAGCCGCCAC-3' and 5'-GTGGCGGCTGGGG GCCTCCGGGCAG-3' for FAM84A-S67A. We transfected NIH3T3 cells with pFLAG-CMV-FAM84A (wild-type FAM84A) or pFLAG-CMV-FAM84A-S38A (mutant FAM84A), and the cells were maintained in the culture media supplemented with $0.9 \mathrm{mg} / \mathrm{ml}$ of Geneticin (Sigma). Three weeks after transfection, we isolated single colonies and established stable clones that express wild-type (3T3-wt FAM84A cells) or mutant FAM84A (3T3-mut FAM84A cells).

Wound-healing assay. Wound-healing migration assay was performed as described elsewhere (13). Briefly, 3T3-wt FAM84A and control (3T3-mock) cells were grown to confluency on 2-well chamber plates coated with fibronectin (Sigma). Cells cultured in media containing $0.1 \%$ FCS were carefully scratched with a plastic pipette tip to form a cross through the confluent monolayers. Cells were further maintained in media containing $10 \%$ FCS. Several wounded areas were marked for orientation and then photographed by phase-contact microscopy at set times ranging from 0 to $72 \mathrm{~h}$.

Transwell migration assay. $2.5 \times 10^{4}$ of 3T3-wt FAM84A or 3T3-mock cells/well were plated onto the top of transwell 
A

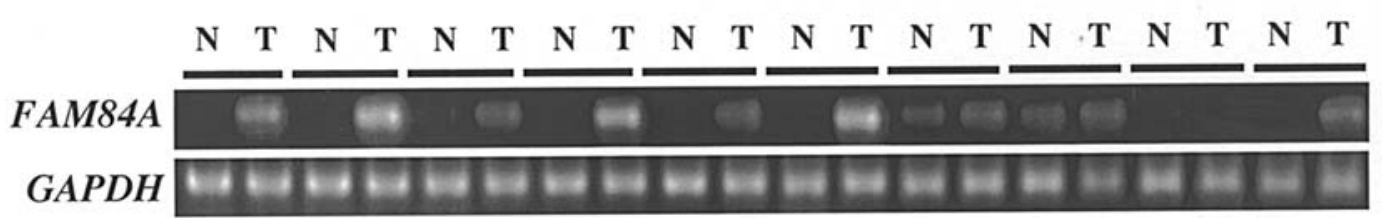

B

FAM84A

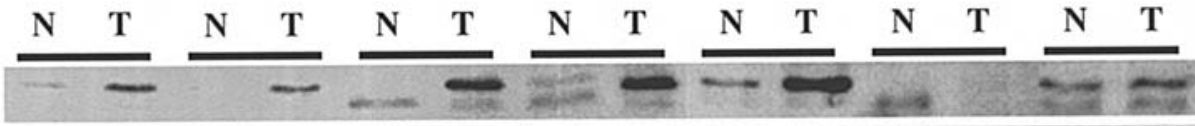

B-actin
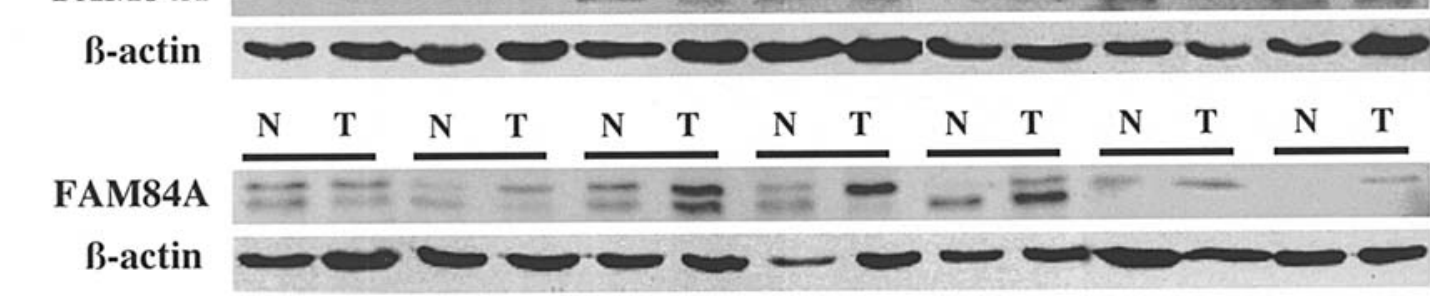

C

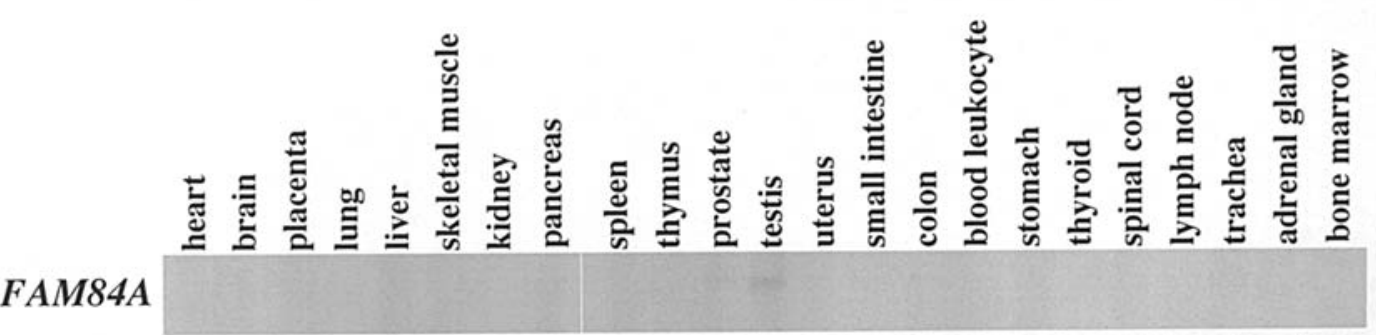

\section{$\beta$-actin}

Figure 1. (A) Semi-quantitative RT-PCR experiment of FAM84A using ten CRC tissues and corresponding normal colonic mucosa. Expression of GAPDH served as a quantitative control. T, tumor tissue; N, normal colon mucosa. (B) Western blot analysis of FAM84A in 14 CRC tissues. T, tumor tissue; N, normal colon mucosa. Expression of $B$-actin served as a quantitative control. (C) Multiple-tissue Northern blot analysis of FAM84A in 23 adult human tissues. Expression of $\beta$-actin served as a quantitative control.

membranes containing pores of $8-\mu \mathrm{m}$ diameter (Becton Dickinson), and cultured in media containing $10 \%$ fetal bovine serum in both upper and lower compartments. After $48 \mathrm{~h}$ of incubation, we removed cells on the upper surface of the filters with a cotton swab, and stained the filters with Giemsa solution for $15 \mathrm{~min}$. Cells that had migrated to the bottom surface of the filters were counted under microscope at magnification $x 400$. The average number of cells in five fields per membrane was counted in triplicate assay.

\section{Results}

Expression of FAM84A in CRC and normal tissues. By means of cDNA microarray analysis, we identified a number of genes whose expression was frequently up-regulated in colorectal cancer cells. We focused on one of them, a gene termed FAM84A/NSE1, encoding a protein that shares $44 \%$ identity with FAM84B/NSE2, another member of FAM84 protein, and $34.5 \%$ with Caenorhabditis elegans Cuticle collagen 12 precursor (Swiss-Prot accession of P20630). Its up-regulation in 7 of 10 colorectal carcinomas was confirmed by subsequent semi-quantitative RT-PCR analysis (Fig. 1A). Our microarray data of other tumors showed that its expression was also elevated in bladder cancers, pancreatic cancers, intrahepatic cholangiocarcinomas, and lung cancers (data not shown). We further carried out Western blot analysis with anti-FAM84A antibody using $14 \mathrm{CRC}$ tissues, and confirmed that FAM84A protein was elevated in 11 of the 14 CRCs compared with the corresponding normal colonic mucosa (Fig. 1B). To further examine expression levels of FAM $84 A$ in human adult normal tissues, we performed multiple-tissue Northern blot analysis using FAM $84 A$ cDNA as a probe, and found no expression of a $4.5-\mathrm{kb}$ transcript in any of the adult tissues examined except in the testis (Fig. 1C).

Expression and subcellular localization of FAM84A protein in CRC lines. We investigated expression of FAM84A in 9 CRC cell lines by Western blot analysis. The analysis demonstrated that SW948 and SW480 cells expressed low levels of 32.5-kDa protein corresponding to the deduced size of FAM84A, and abundant 33.5-kDa protein (Fig. 2A). Immunocytochemical staining of SW948 cells detected a quite unique pattern of FAM84A protein; its staining was observed in the sub-plasma membrane region, but it was limited to the region where cells did not have contact with neighboring cells (Fig. 2B).

Phosphorylation of FAM84A. Since we identified two sizes (32.5 and $33.5 \mathrm{kDa}$ ) of the FAM84A protein by Western blot analysis, we considered some modification of FAM84A. To examine a possible phosphorylation of the protein, we performed a $\lambda$-phosphatase assay, and found the disappearance of the larger molecular protein by treatment of $\lambda$-phosphatase 
A

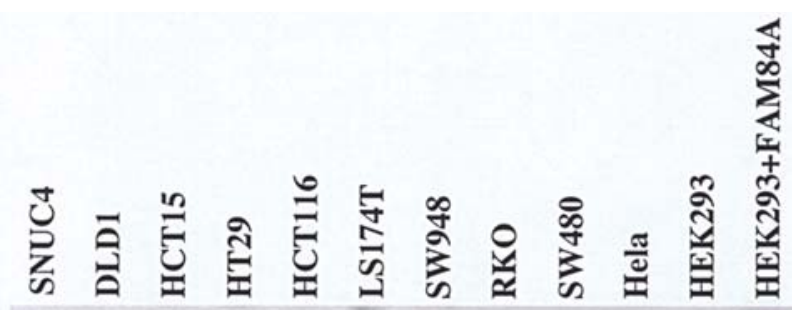
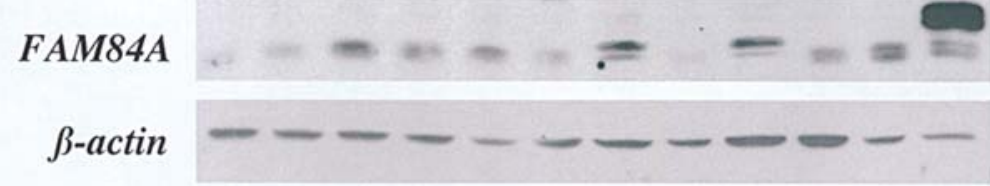

B

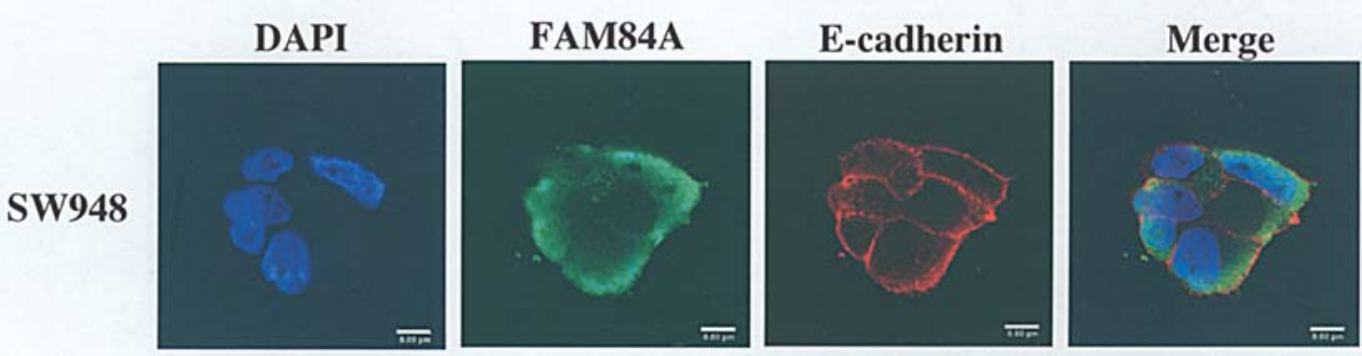

Figure 2. (A) Western blot analysis of FAM84A in cancer cell lines. HEK293 cells transfected with pFLAG-CMV-FAM84A expressed exogenous FLAG-tagged FAM84A protein and endogenous FAM84A protein. (B) Subcellular localization of FAM84A. Immunocytochemical staining of FAM84A protein in SW948 cells. Cells were fixed and stained with anti-FAM84A and anti-E-cadherin antibodies. For fluorescent immunostaining, antibodies were stained with anti-rabbit secondary antibody conjugated to Alexa Fluor 488 and anti-mouse secondary antibody conjugated to Alexa Fluor 594. Nuclei were counterstained in blue with DAPI. Magnification x600. Bars, $8 \mu \mathrm{m}$.

A

\section{$\lambda$-PPase \\ IB: $\alpha$ FLAG}

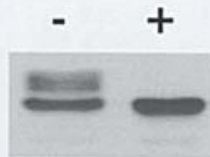

C

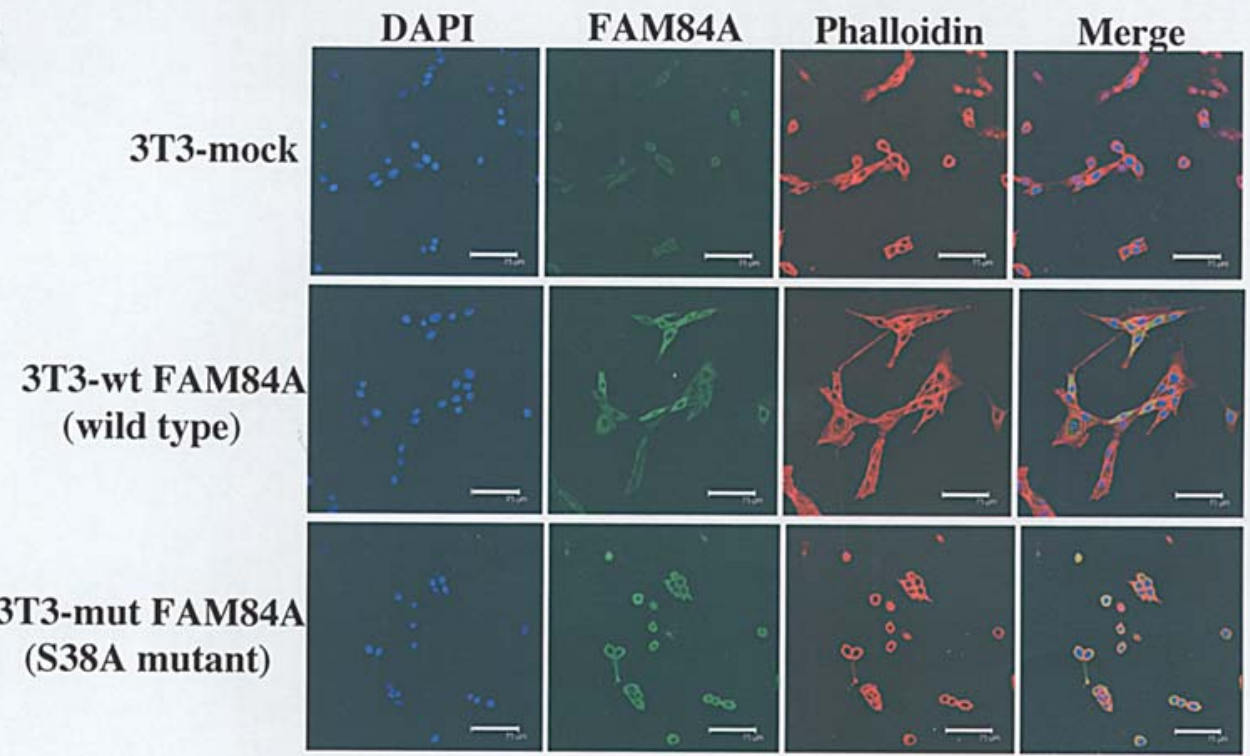

Figure 3. (A) Phosphorylation of FAM84A in vivo. Extracts from HEK293 cells expressing FLAG-tagged wild-type FAM84A protein were treated with or without $\lambda$-phosphatase. (B) Phosphorylation of wild-type and mutant forms of FAM84A (FAM84A-S38A and FAM84A-S67A). (C) Morphology of 3T3-wt FAM84A cells and 3T3-mut FAM84A cells. Cells were fixed and stained with anti-FAM84A antibody (second panel) and Phalloidin (third panel). Merged images are shown in the right panel. Bars, $75 \mu \mathrm{m}$. 
A

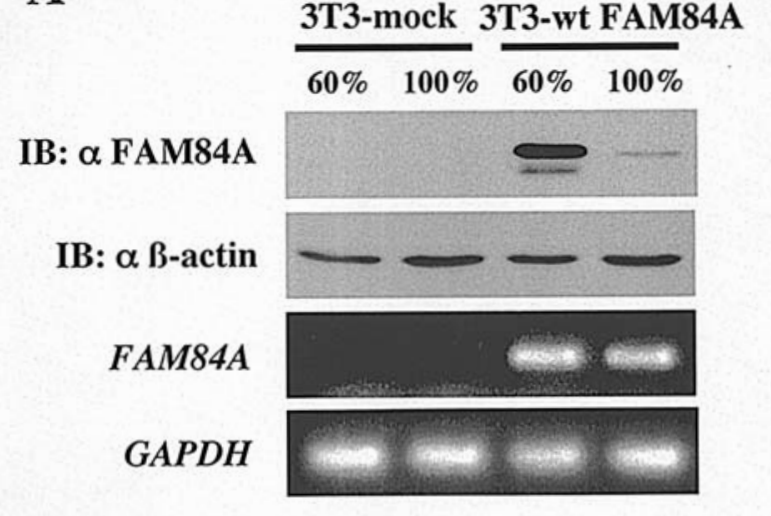

B

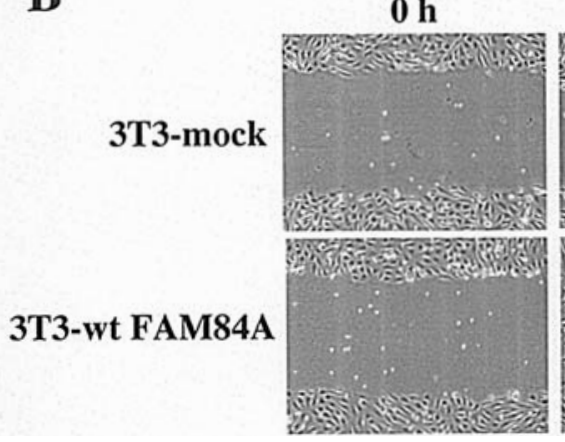

$24 \mathrm{~h}$

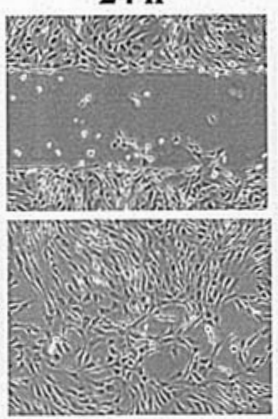

C

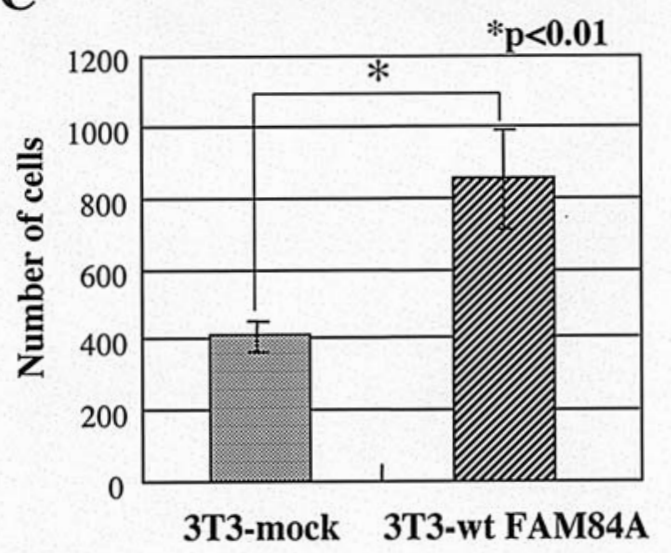

$48 \mathrm{~h}$

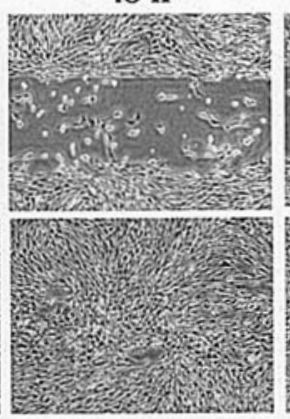

$72 \mathrm{~h}$

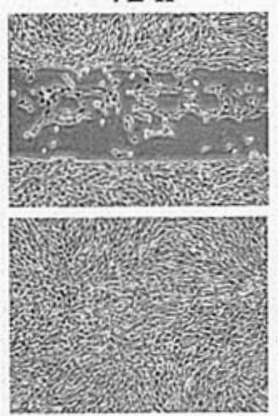

Figure 4. (A) Posttranscriptional regulation of FAM84A protein dependent on density of cultured cells. Expression of FAM84A protein was analyzed by Western blot analysis at 60 and $100 \%$ cell densities (upper panel). Expression of $\beta$-actin served as a quantitative control. Expression of FAM84A transcript was measured by semi-quantitative RT-PCR (3rd panel). (B) Cell migration was analyzed by wound-healing assay. Representative images of migrating cells were captured 24, 48 and $72 \mathrm{~h}$ after the scratch. (C) Transwell assays of 3T3-wt FAM84A cells. The results were the average number of cells in 5 fields per membrane at $48 \mathrm{~h}$.

(Fig. 3A), indicating the phosphorylation of FAM84A. An algorithm, Motif scan (http://scansite.mit.edu/), predicted two putative phosphorylation sites, serine 38 and serine 67 , in FAM84A. We expressed two forms of mutant plasmids expressing the two proteins, in which serine 38 or serine 67 was replaced to alanine (pFLAG-CMV-FAM84A-S38A and pFLAG-CMV-FAM84A-S67A), in HEK293 cells. Subsequent Western blot analysis revealed no phosphorylation of FAM84A-S38A while both FAM84A itself and FAM84AS67A showed both phosphorylated and unphosphorylated forms (Fig. 3B). These data indicate that serine 38 is a target site for phosphorylation of FAM84A.

Involvement of FAM84A in morphology of cells. To clarify the function of FAM84A, we carried out colony formation assay using NIH3T3 cells. However, we observed no significant difference in the number of colonies between cells expressing wild-type FAM84A and control cells (data not shown). We further established NIH3T3 cells that stably expressed exogenous wild-type FAM84A (3T3-wt FAM84A) and those expressing FAM84A-S38A (3T3-mut FAM84A). Consistent with the result of colony formation assay, growth of 3T3-wt FAM84A cells was similar to that of 3T3-mock or NIH3T3mut FAM84A cells (data not shown). Interestingly 3T3-wt FAM84A cells showed a spindle-like shape, as did the parental NIH3T3 or 3T3-mock cells, but 3T3-mut FAM84A cells expressing the unphosphorylated form revealed a round shape
(Fig. 3C), indicating that FAM84A plays some role in the cell morphology, and that phosphorylation at serine 38 is essential in this regard.

Post-transcriptional regulation of FAM84A protein. Interestingly, we found that expression of FAM84A protein was drastically altered by the density of cultured cells; 3T3-wt FAM84A cells expressed abundant FAM84A protein when they were cultured in growing conditions at $60 \%$ density of cells, but the expression was markedly downregulated in static conditions at $100 \%$ confluent density (Fig. 4A, upper panel). Since expression of FAM84A transcripts was similar at 60 and $100 \%$ density of cells, the decreased expression should result from posttranscriptional regulation such as increased protein degradation or suppressed translation (Fig. 4A). Expression of FAM84A-S38A protein was also reduced when the cells were at $100 \%$ confluent density compared to $60 \%$ confluent density (data not shown). Therefore, phosphorylation of FAM84A serine 38 may not be involved in the posttranscriptional modulation.

Effect of FAM84A on motility of cancer cells. The unique subcellular localization, altered FAM84A expression by the density of cultured cells, and its involvement in morphology tempted us to hypothesize that FAM84A might have some function in motility or migration of cancer cells. We therefore performed wound-healing assay using 3T3-mock and 3T3-wt 
FAM84A cells. As shown in Fig. 4B, 3T3-wt FAM84A cells migrated rapidly, and filled in the wound significantly faster than 3T3-mock cells, implying that FAM84A is associated with migration of cells. To confirm the effect of FAM84A on migration, we additionally carried out a transwell assay. Consistent with the data of wound-healing assay, we observed an augmented number of migrated 3T3-wt FAM84A cells through the pores of chambers compared to control cells at $48 \mathrm{~h}$ of culture (Fig. 4C). These data indicated that FAM84A plays a role in cellular migration, because both cells showed a similar proliferation rate as described previously.

\section{Discussion}

FAM84A shares $34.5 \%$ similarity in amino acids with $C$. elegans Cuticle collagen 12 precursor (Swiss-Prot accession of P20630), 26.9\% with Mus musculus Collagen $\alpha$ I chain precursor (Swiss-Prot accession of P11087), and 28.3\% with human collagen, type X, $\alpha$ I (Swiss-Prot accession of Q03692). Since these proteins are components of extracellular matrices (ECM), we considered a possible role of FAM84A in the regulation of cell-microenvironment interaction. Integrins, key players of the interaction between cell and ECM, regulate cell adhesion to ECM by a formation of focal adhesion, which contains associated proteins including $\alpha$-actinin, vinculin, tensin, paxillin, FAK, and CAS $(14,15)$. These molecules not only link integrins with the cytoskeleton, they also mediate signals from ECM to cells as well as those from cells to integrins. Therefore, FAM84A may play a role in the interaction between cell and ECM. FAM84B/NSE2, showing 44\% identity to FAM84A in amino acids, is reported to localize in cellular membrane and associate with $\alpha$-catenin (16). We therefore tested whether FAM84A also interacts with $\alpha$-catenin or not, but failed to show their association in vivo. Since the subcellular localization of FAM84A protein is different to that of FAM84B, we suspect that FAM84A is not directly involved in cell-cell adhesion.

In this study, we revealed that cancer cells accumulated FAM84A as both phosphorylated and unphosphorylated forms, and that exogenous expression of wild-type FAM84 did not enhance the growth of cells but increased cell motility. In agreement with this finding, we found that FAM84A localized in the sub-cytoplasmic membrane region in a part where cells have no contact with neighboring cells. Because cells expressing a dephosphorylated form of FAM84A showed altered cellular morphology, phosphorylation of serine 38 may be essential for morphogenesis. Therefore, FAM84A may mediate signals associated with cytoskeletal remodeling and cellular migration. Recent studies suggest an interplay between remodeling of the cytoskeleton and migration. For example, cytoplasmic molecules such as Rho, Rac1, and cdc42 not only modulate remodeling of the cytoskeleton but also play a role in cell motility. As central regulators of cytoskeletal dynamics, Rho family GTPases stimulate the formation of actin stress fibers and mature elongated focal adhesion. Cdc42 stimulates actin polymerization and the formation of thin membrane protrusions called filopodia. Rac1 stimulates the processes of membrane ruffling and cell spreading that result in the formation of actin-rich lamellipodial protrusions (11). Therefore, FAM84A may be involved in the modulation of the signal transduction pathway played by Rho family GTPases.

Invasion involves multiple events associated with molecules that are involved in cell-cell adhesion, cellular motility, interaction between cell and microenvironment, such as inactivation of cell-cell adhesion by decreased expression of E-cadherin (17), increased cell motility by activation of RhoC $(18,19)$, and augmented proteolysis of extracellular matrices by elevated expression of matrix metalloproteinases. Further studies of its interacting protein(s) and modification enzyme(s) will unveil mechanisms of cellular motility associated with FAM84A.

Since FAM84A is enhanced in bladder cancer, pancreatic cancer, cholangiocarcinoma (CCC), lung cancer and colorectal cancers, FAM84A may be involved in common mechanisms of carcinogenesis. Therefore, a profound understanding of the mechanisms played by FAM84A in carcinogenesis may contribute to the development of novel diagnostic and therapeutic approaches for a wide range of human tumors.

\section{Acknowledgments}

We are grateful to Dr Ryuji Hamamoto, Satoshi Matsushima and Dr Michihiro Sakai for helpful discussion. This work was supported in part by Research for the Future Program Grant (00L01402) from Japan Society for the Promotion of Science.

\section{References}

1. Jemal A, Tiwari RC, Murray T, Ghafoor A, Samuels A, Ward E, Feuer EJ and Thun MJ: American Cancer Society: Cancer stastistics, 2004. CA Cancer J Clin 54: 8-29, 2004.

2. Tamakoshi K, Wakai K, Kojima M, Watanabe Y, Hayakawa N, Toyoshima H, Yatsuya H, Kondo T, Tokudome S, Hashimoto S, Suzuki K, Ito Y and Tamakoshi A: JACC Study Group: a prospective study of body size and colon cancer mortality in Japan. The JACC Study. Int J Obes Relat Metab Disord 28: 551-558, 2004.

3. Sakamoto J, Ohashi Y, Hamada C, Buyse M, Burzykowski T and Piedbois P: Meta-Analysis Group of the Japanese Society for Cancer of the Colon and Rectum; Meta-Analysis Group in Cancer: efficacy of oral adjuvant therapy after resection of colorectal cancer: 5-year results from three randomized trials. J Clin Oncol 22: 484-492, 2004.

4. Weitz J, Koch M, Debus J, Hohler T, Galle PR and Buchler MW: Colorectal cancer. Lancet 365: 153-165, 2005.

5. Heijstek MW, Kranenburg O and Borel Rinkes IH: Mouse models of colorectal cancer and liver metastases. Dig Surg 22: 16-25, 2005.

6. Saha D, Roman C and Beauchamp RD: New strategies for colorectal cancer prevention and treatment. World J Surg 26: 762-766, 2002.

7. De la Chapelle A: Genetic predisposition to colorectal cancer. Nat Rev Cancer 4: 769-780, 2004.

8. Feinberg AP and Tycko B: The history of cancer epigenetics. Nat Rev Cancer 4: 143-153, 2004.

9. Robertson KD: DNA methylation and human disease. Nat Rev Genet 6: 597-610, 2005.

10. Cui H, Cruz-Correa M, Giardiello FM, Hutcheon DF, Kafonek DR, Brandenburg S, Wu Y, He X, Powe NR and Feinberg AP: Loss of IGF2 imprinting: a potential marker of colorectal cancer risk. Science 299: 1753-1755, 2003.

11. Geho DH, Bandle RW, Clair T and Liotta LA: Physiological mechanisms of tumor-cell invasion and migration. Physiology 20: 194-200, 2005.

12. Lin YM, Furukawa Y, Tsunoda T, Yue CT, Yang KC and Nakamura Y: Molecular diagnosis of colorectal tumors by expression profiles of 50 genes expressed differentially in adenomas and carcinomas. Oncogene 21: 4120-4128, 2002. 
13. Malinda KM, Sidhu GS, Mani H, Banaudha K, Maheshwari RK, Goldstein AL and Kleinman HK: Thymosin beta 4 stimulates directional migration of human umbilical vein endothelial cells. FASEB J 11: 472-481, 1997.

14. Guo W and Giancotti FG: Integrin signalling during tumour progression. Nat Rev Mol Cell Biol 5: 816-826, 2004.

15. Izard T, Evans G, Borgon RA, Rush CL, Bricogne G and Bois P: Vincullin activation by talin though helical bundle conversion. Nature 427: 171-175, 2004.

16. Adam PJ, Boyd R, Tyson KL, Fletcher GC, Stamps A, Hudson L, Poyser HR, Redpath N, Griffiths M, Steers G, Harris AL, Patel S, Berry J, Loader JA, Townsend RR, Daviet L, Legrain P, Parekh R and Terrett JA: Comprehensive proteomic analysis of breast cancer cell membranes reveals unique proteins with potential roles in clinical cancer. J Biol Chem 278: 6482-6489, 2003.
17. Bjorklund M and Koivunen E: Gelatinase-mediated migration and invasion of cancer cells. Biochim Biophys Acta 1755: 37-69, 2005

18. Hakem A, Sanchez-Sweatman O, You-Ten A, Duncan G, Wakeham A, Khokha R and Mak TW: RhoC is dispensable for embryogenesis and tumor initiation but essential for metastasis. Genes Dev 19: 1974-1979, 2005.

19. Wheeler AP and Ridley AJ: Why three Rho proteins? RhoA, RhoB, RhoC and cell motility. Exp Cell Res 301: 43-49, 2004. 